\title{
Influência do dano mecânico de impacto e compressão sobre a respiração de maçãs cultivares Fuji Suprema e Royal Gala
}

\section{Influence of impact and compression mechanical damage on respiration of Fuji Suprema and Royal Gala apples}

\author{
Cândida Raquel Scherrer Montero ${ }^{1 *}$; Liege Cunha dos Santos²; \\ Cristiane Salete Andreazza ${ }^{3}$; Renar João Bender ${ }^{4}$
}

\begin{abstract}
Resumo
A intensidade da respiração celular está relacionada com o potencial de armazenamento nos produtos hortícolas; no caso de maçãs, a principal modificação é a metabolização dos ácidos orgânicos. Danos mecânicos são freqüentes na pós-colheita de frutos, e estas forças externas podem acelerar o padrão respiratório destes, acarretando em perdas posteriores. Os objetivos desse trabalho foram avaliar os efeitos de danos mecânicos por impactos e compressões sobre o padrão respiratório de maçãs Fuji Suprema e Royal Gala. Os experimentos consistiram de cinco tratamentos com três repetições e seis frutos por repetição. Após a aplicação dos tratamentos, os frutos foram colocados em frascos vedados, mantidos à temperatura ambiente onde foram realizadas as determinações do $\mathrm{CO}_{2}\left(\mathrm{ml} \mathrm{CO}_{2} \mathrm{~kg}^{-1} \mathrm{~h}^{-1}\right) \mathrm{com}$ analisador equipado com detector de zircônio. As leituras ocorreram a 0, 1, 2, 4, 6, 24, 48 e 168 horas após a instalação e os dados foram submetidos à análise de regressão. Houve um aumento da respiração após a aplicação dos tratamentos. O maior incremento, para maior parte dos tratamentos, foi observado nas primeiras seis horas, com posterior redução nas taxas de respiração após 24 horas. As curvas de regressão da intensidade respiratória em função dos tratamentos evidenciam que o incremento nas taxas respiratórias está associado com a intensidade das forças mecânicas aplicadas. Os incrementos nas taxas respiratórias foram maiores em maçãs Gala do que Fuji e as forças de impacto produzem maior efeito sobre as taxas respiratórias que as de compressão.
\end{abstract}

Palavras-chave: Malus domestica $\mathrm{B}$, perecibilidade, injúria mecânica, respiração celular

\begin{abstract}
The intensity of cell respiration is related to the storage potential of a horticultural product. In the case of apples the main modification during storage is the metabolization of organic acids. Mechanical damage is frequent in fruit post harvest handling and the external forces involved can accelerate the respiratory pattern of fruits leading to further losses. The objectives of this work were to evaluate the effects of mechanical damage by impact and compressing on the respiratory pattern of Fuji Suprema and Royal Gala apples. The experiments consisted of five treatments with three replications of six fruit each. After the treatment application, fruit were set in closed flasks and maintained in room temperature during the $\mathrm{CO}_{2}$ determination $\left(\mathrm{ml} \mathrm{CO}_{2} \mathrm{~kg}^{-1} \mathrm{~h}^{-1}\right)$. The measurements were made with a $\mathrm{CO}_{2}$ analyzer equipped with a zircon detector. The readings were taken at $0,1,2,4,6,24,48$ and 168 hours after the treatment and data
\end{abstract}

\footnotetext{
${ }^{1}$ Engenheiro Agrônomo MSc. Estudante de Pós-Graduação em Fitotecnia, Faculdade de Agronomia, Universidade Federal do Rio Grande do Sul - UFRGS, bolsista Capes. E-mail: candidaraquel@gmail.com

${ }^{2}$ Estudante de Graduação em Agronomia, UFRGS, bolsista CNPq. E-mail: liegesantos@ibest.com.br

${ }^{3}$ Estudante de Graduação em Agronomia, UFRGS, bolsista CNPq. E-mail: liegesantos@ibest.com.br

${ }^{4}$ Professor Dr. no Departamento de Horticultura e Silvicultura, Faculdade de Agronomia, UFRGS, bolsista CNPq. E-mail: rjbe@ ufrgs.br

* Autora para correspondência
} 
were submitted to a regression analysis. There was an increase in fruit respiration after the treatment application. The respiratory profiles along the evaluated period show increase of the respiratory rate after the treatment application. The greatest increase for most treatments occurred within the first six hours after the treatment, with posterior reduction. The regression curves of the respiratory rate in function of the treatment applied showed there is an increment in the respiratory rates associated with the intensity of applied forces. The increments of respiratory rates were greater for Gala than Fuji apples and impacts produce a greater effect over the respiration than compression forces.

Key words: Malus domestica Borkh, perishability, mechanical injury, cellular respiration

\section{Introdução}

A respiração celular é o processo metabólico pelo qual se produz energia química na forma de ATP usada nas reações vitais internas e nos processos de síntese e manutenção celular (SAQUET; STREIF; BANGERTH, 2000). As maçãs, durante o armazenamento, metabolizam preferencialmente ácidos orgânicos, cujo conteúdo poderá ser reduzido até pela metade dependendo das condições de armazenamento (SAQUET; STREIF, 2002). A intensidade respiratória é usada para predizer o potencial de armazenamento de um produto, pois quanto mais elevada for a respiração, menor será tempo de conservação e vice-versa (LAU, 1985; PATTERSON, 1989).

Danos mecânicos são extremamente freqüentes no manuseio de inúmeros frutos durante a sua pós-colheita. Os danos mecânicos são definidos como deformações plásticas, rupturas superficiais e destruição dos tecidos vegetais provocados por forças externas (SANCHES; DURIGNA; DURIGAN, 2008). De maneira geral, é aceitável afirmar que danos mecânicos aumentam as taxas respiratórias de frutos induzindo a perdas qualitativas posteriores. Nesse sentido, alguns estudos já foram realizados com inúmeras espécies frutíferas: goiabas (MATTIUZ; DURIGAN, 2001), maçãs cultivar Gala (STEFFENS et al., 2008), peras (AGAR; MICHAN, 2000), damascos (DeMARTINO et al., 2006), melões Cantaloupe (MacLEOD; KADER; MORRIS, 1976), lima ácida Tahiti (DURIGAN; MATIIUZ; DURIGAN, 2005) e cerejas (BURTON; SCHULTE-PASON, 1987). No entanto, poucos são os estudos que comparam os distintos tipos de danos mecânicos ou mencionam as intensidades de danos capazes de produzir efeitos na respiração de maçãs. Além disso, é importante salientar que há conclusões distintas sobre o tema, muitas vezes relacionadas a diferentes metodologias de avaliação do processo de respiração. A maior parte dos estudos avalia dias após o tratamento de dano mecânico, como o trabalho de Saquet e Streif (2002), em maçãs. Além disso, há dados controversos a este respeito dos efeitos dos danos mecânicos sobre a respiração celular, sendo que alguns verificaram incrementos na respiração enquanto outros não observaram alteração no metabolismo.

Nesse sentido, o objetivo deste trabalho foi avaliar os efeitos dos danos mecânicos de impacto e compressão sobre o padrão respiratório de maçãs Fuji Suprema e Royal Gala.

\section{Material e Métodos}

As maçãs das cultivares Royal Gala e Fuji Suprema foram colhidas no município de Vacaria e trazidas ao laboratório de Fisiologia Pós-colheita no mesmo dia da colheita. Os experimentos foram instalados de um a dois dias após a colheita e avaliados por sete dias. Foram aplicados cinco tratamentos, cada um com três repetições de cinco a seis frutos dependendo do tamanho dos frutos colhidos para cada cultivar.

Os tratamentos de dano mecânico aplicados nos frutos foram de dois tipos: impactos $\mathrm{e}$ compressões.

Os impactos consistiram em choques das maçãs contra uma superfície rígida de cerâmica por duas vezes de alturas que variaram de 20 a $160 \mathrm{~cm}$. 

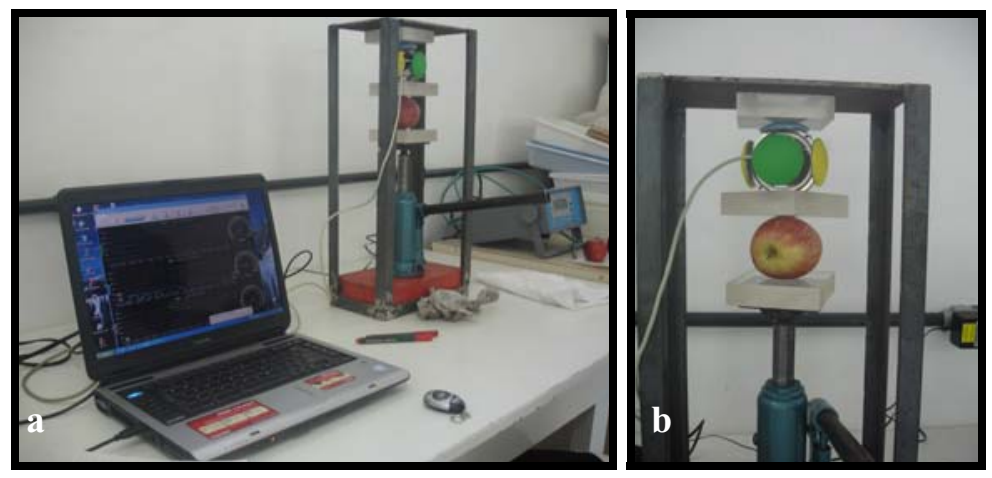

Figura 1. Equipamento de aplicação de compressões constituído por um macaco hidráulico, duas placas acrílicas e a esfera instrumentada conectada a um computador (a) detalhe da aplicação do tratamento em uma maçã (b).

As compressões foram aplicadas com auxílio de um equipamento constituído por um macaco hidráulico fixo em uma placa metálica rígida (Figura 1). Os frutos foram colocados um a um entre a prensa do equipamento em série com a esfera instrumentada. A esfera instrumentada utilizada nestes ensaios é parte de um sistema de análise formado por quatro partes: uma esfera de alumínio composta por três anéis equipados com extensiômetros (dispositivos sensores das deformações mecânicas); uma placa de circuito impresso colada nos extensiômetros localizada no interior da esfera, junto com os amplificadores de instrumentação para extensiômetros; uma unidade externa contendo um sistema de aquisição de dados para a conversão dos sinais analógicos oriundos da esfera em sinais digitais para comunicação com o computador por software específico. De acordo com Muller, Brito e Bender (2008) a EI é calibrada para medir forças estáticas. Desta forma, à medida que o macaco hidráulico é acionado comprimindo simultaneamente as maçãs e a esfera, as forças de compressão são identificadas por um software especialmente desenvolvido para adquirir os dados percebidos pela esfera instrumentada. Compressões de $40 \mathrm{~N}, 80 \mathrm{~N}, 160 \mathrm{~N}$ e $330 \mathrm{~N}$ foram aplicadas sobre as maçãs.

Após a aplicação do tratamento, as maçãs foram colocadas em frascos de vidro que foram vedados e mantidos à temperatura ambiente $\left(20^{\circ} \mathrm{C} \pm 2^{\circ} \mathrm{C}\right)$ durante os períodos de determinação da produção de $\mathrm{CO}_{2}$. Essas determinações ocorreram 0, 1, 2, 4, 6, 24, 48 e 168 horas após o tratamento. A produção de $\mathrm{CO}_{2}$ das maçãs expressa em $\mathrm{ml} \mathrm{CO}$ $\mathrm{kg}^{-1} \mathrm{~h}^{-1}$, foi avaliada com auxílio de um analisador de $\mathrm{CO}_{2}$ equipado com detector de zircônia (marca Climasul), o qual era diariamente calibrado com um padrão de $\mathrm{CO}_{2}$ com concentração conhecida.

Foram realizados quatro ensaios, sendo dois de impacto e dois de compressão para cada cultivar avaliada, cada ensaio com quatro intensidades de danos mecânicos. O delineamento foi completamente casualizado e os dados foram submetidos à análise de regressão.

\section{Resultados}

Ocorreu incremento da taxa respiratória após os tratamentos de dano mecânico de impacto e compressão aplicados em ambas as cultivares (Figuras 2 a 5). Há um incremento maior no período entre quatro e seis horas após o tratamento, e uma redução nas taxas de produção de $\mathrm{CO}_{2}$ posteriores a 24 horas após o tratamento. Há, no entanto, uma exceção: o tratamento de queda sobre superfície rígida a $160 \mathrm{~cm}$. Neste tratamento ainda pode ser determinado um incremento significativo na produção de $\mathrm{CO}_{2}$ posterior ao período de 24 horas de aplicação do tratamento. 

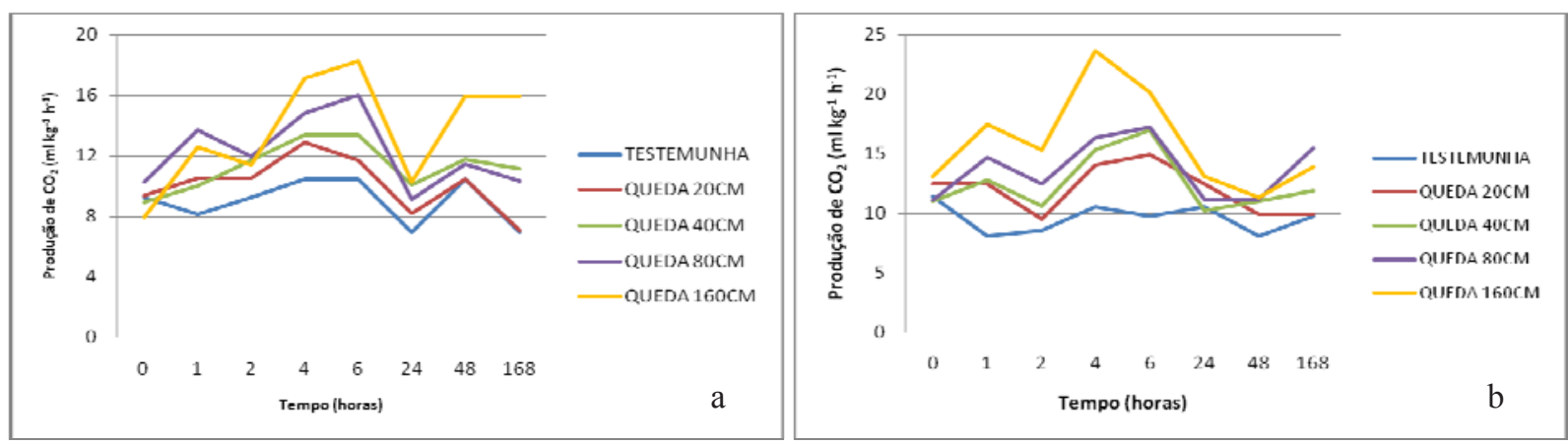

Figura 2. Respiração de maçãs cultivares Fuji Suprema (a) e Royal Gala (b) submetidas a danos mecânicos de impacto ao longo de 7 dias.
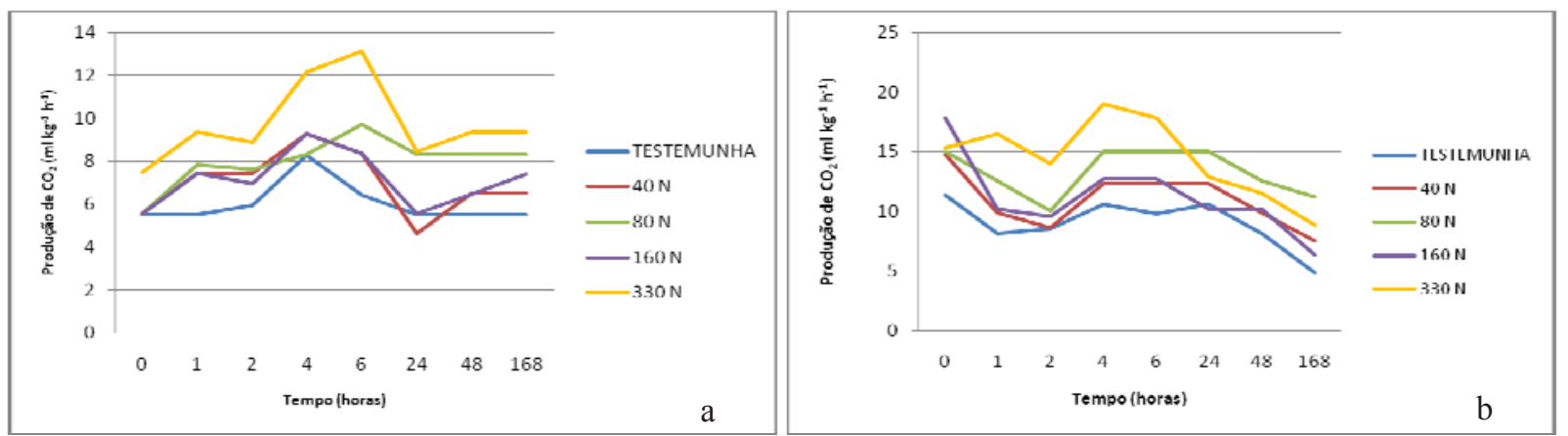

Figura 3. Respiração de maçãs cultivares Fuji Suprema (a) e Royal Gala (b) submetidas a danos mecânicos de compressão ao longo de 7 dias.

Para a maioria dos tratamentos de impacto e compressão, o incremento nas taxas respiratórias ocorre nas primeiras horas após o tratamento, e não dias após o mesmo. É possível visualizar a resposta dos tratamentos de acordo com a intensidade dos mesmos pela separação das curvas no caso dos frutos expostos a forças de impacto (Figura 2). Nesta situação, as maçãs expostas ao tratamento

de queda a uma altura maior são aquelas que apresentam maiores taxas respiratórias ao longo do experimento. Já no caso dos tratamentos de compressão, essa tendência não é vista com tanta clareza, mas mesmo assim, o tratamento de maior intensidade produziu o maior efeito sobre as taxas respiratórias (Figura 3).
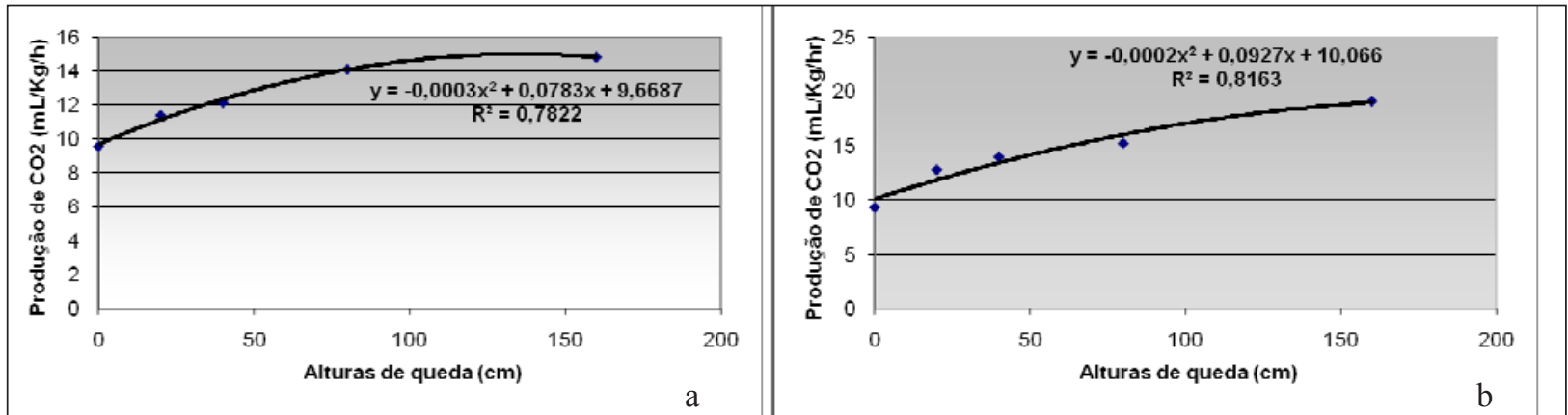

Figura 4. Produção de $\mathrm{CO}_{2}$ em maçãs Fuji Suprema (a) e Royal Gala (b) em função do grau do dano mecânico de impacto. 


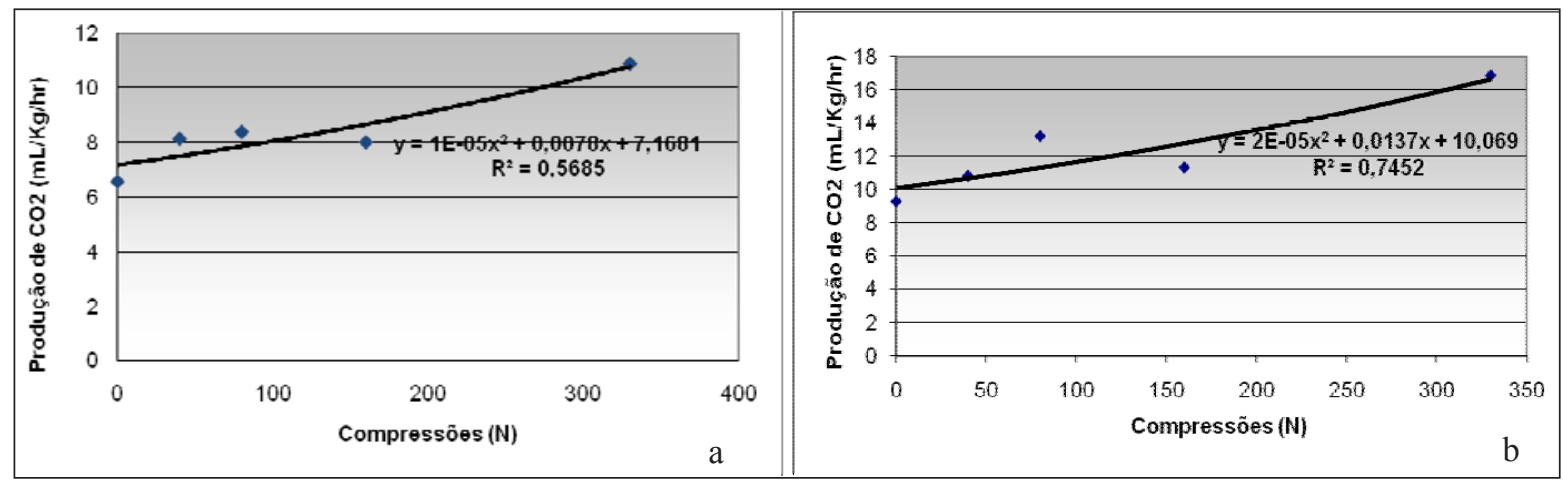

Figura 5. Produção de $\mathrm{CO}_{2}$ em maçãs Fuji Suprema (a) e Royal Gala (b) em função do grau do dano mecânico de compressão.

Os efeitos da intensidade dos tratamentos podem ser mais bem entendidos pela análise de regressão em ambos os casos (Figuras 4 a 5). As curvas de resposta da respiração aos danos mecânicos provocados por impacto e compressão são regressões polinomiais. Em ambas as cultivares há efeito dos danos mecânicos sobre a intensidade respiratória, com incremento dessas taxas em função da intensidade do dano provocado no fruto. As taxas respiratórias das maçãs submetidas a impactos tiveram um incremento que variou de $19 \%$ a $55 \%$ do tratamento menos ao mais intenso para maçãs cv. Fuji, e de $38 \%$ a $100 \%$ para a cv. Gala. No caso das compressões, o incremento variou de $24 \%$ a $66 \%$ na cv. Fuji do tratamento menos ao mais intenso, e de $16 \%$ a $82 \%$ na cv. Gala. Mesmo assim, comparandose a resposta das taxas respiratórias dos danos de impacto com os de compressão percebe-se que, em termos absolutos, as taxas chegam a valores mais elevados para os frutos quando submetidos a impactos em comparação a compressões, em ambas as cultivares.

Comparando as cultivares estudadas, a Royal Gala, que naturalmente apresenta taxas respiratórias mais elevadas do que a Fuji, aquela também apresentou incrementos maiores nestas taxas em função dos danos aplicados.

\section{Discussão}

Taxas respiratórias são indicadores de atividade metabólica de hortaliças, podendo indicar quão rápido senescem (BLANKE, 1991). De um modo geral, frutos e outros produtos hortícolas com altas taxas respiratórias tendem a ter vida de prateleira curta, enquanto que aqueles com baixas taxas respiratórias tendem a ter maior vida de prateleira (KADER, 1985; BLANKE, 1991; WILLS et al., 1998). Os órgãos vegetais submetidos a vibrações e danos mecânicos em geral aumentam suas taxas respiratórias em comparação com testemunhas sem injúrias (PISARCZYK, 1982; SALVEIT, 1982; MAO et al., 1995). No trabalho de Steffens et al. (2008) não ocorreram respostas significativas em função de danos mecânicos de impacto a uma altura de queda de $20 \mathrm{~cm}$. Ao contrário do proposto por Steffens et al. (2008), o presente trabalho evidenciou incremento nas taxas respiratórias de maçãs em função dos tratamentos de força mecânica aplicados. No entanto, é bom salientar que foram trabalhos distintos. Neste avaliouse a intensidade respiratória ao longo do tempo incluindo curtos períodos após o tratamento, dois tipos de danos mecânicos, impactos e compressões e quatro intensidades de dano mecânico, enquanto os autores do trabalho mencionado acima testaram duas quedas a $20 \mathrm{~cm}$ e as avaliações da respiração 
ocorreram ao longo de dias após o tratamento. Tratamentos menos intensos aliados a avaliações em períodos mais longos após a exposição ao dano mecânico poderiam ser a razão pela qual os mesmos não encontram respostas significativas em termos de incremento nas taxas respiratórias das maçãs, uma vez que o presente trabalho mostra acréscimo na taxa respiratória, principalmente nas primeiras horas após o tratamento, seguida de posterior redução após vinte e quatro horas.

Por outro lado, outros trabalhos já encontraram respostas de incremento nas taxas respiratórias em função de danos mecânicos em outros produtos hortícolas como peras (AGAR; MICHAN, 2000), goiabas (MATTIUZ; DURIGAN, 2001), mandioquinha salsa (HENZ et al., 2005) e lima ácida Tahiti (DURIGAN; MATIIUZ; DURIGAN, 2005).

Hendges et al. (2008), estudando danos mecânicos de impacto em maçãs das cultivares Royal Gala e Pink Lady, encontraram redução das taxas respiratórias com aumento da intensidade dos danos mecânicos na avaliação do terceiro dia após o tratamento. No presente trabalho ocorreram reduções nas taxas respiratórias das maçãs em vinte e quatro horas da aplicação do tratamento para todos os tratamentos de danos mecânicos. Uma tendência similar de redução para os tratamentos de $160 \mathrm{~cm}$ de queda e $330 \mathrm{~N}$ de compressão, obtida para cultivar Royal Gala (Figuras $2 b$ e $3 b$ ). No caso da cultivar Fuji, tal efeito não ocorreu. Hendges et al. (2008) avaliaram as taxas respiratórias ao longo de 3 a 15 dias após a aplicação do tratamento.

\section{Conclusões}

Há incremento na taxa respiratória de maçãs submetidas a danos mecânicos por compressão e impacto. O incremento é influenciado pela intensidade da força aplicada e ocorre nas primeiras horas após o tratamento com posterior redução do efeito.
Forças dinâmicas, como os impactos, produzem maior efeito absoluto sobre a respiração de maçãs que forças estáticas, como as de compressão.

As maçãs da cultivar Gala sofrem mais com danos mecânicos que as da cultivar Fuji, visto que têm suas taxas respiratórias mais aceleradas.

\section{Agradecimentos}

À Capes e ao CNPq pelas bolsas que financiaram a pesquisa. A empresa Schio pela doação das maçãs para estes experimentos. A Loana, Viviane e Leusiane, por permitirem a colheita junto ao local de seus experimentos e pelo excelente convívio e troca de idéias durante o curso dos experimentos.

\section{Referências}

AGAR, I. T.; MICHAN, E. J. Commercial handling influences quality and ripening of bartlett pears. California Agriculture, Oakland, v. 54, n. 3, p. 34-37, 2000.

BLANKE, M. M. Respiration of apple and avocado fruit. Postharvest News and Information, United Kingdom, v. 2, n. 6, p. 429-436, 1991.

BURTON, C. L.; SCHUlTE-PASON, N. L. Carbon dioxide as an indicator of fruit impact damage. HortScience, Alexandria, v. 22, n. 2, p. 281-282, 1987.

DeMARTINO, G.; VIZOVITIS, K.; BOTONDI, R.; BELLINCONTRO, A.; MENCARELLI, F. 1-MCP controls ripening induced by impact injury on apricots by afeectin SOD and POX activities. Postharvest Biology and Technology, Amsterdam, v. 39, n. 1, p. 369-372, 2006.

DURIGAN, M. F. B.; MATIIUZ, B. H; DURIGAN, J. F. Injúrias mecânicas na qualidade pós-colheita de lima ácida "Tahiti" armazenada sob condições ambiente. Revista Brasileira de Fruticultura, Jaboticabal, v. 27, n. 3, p. 369-372, 2005.

HENDGES, M. V.; STEFFENS, C. A.; ANTONIOLLI, L. R.; AMARANTE, C. V. T. do; ZANARDI, O. Z. Taxas respiratórias e de produção de etileno em maçãs 'Royal Gala `e `Pink Lady` submetidas a diferentes níveis de dano mecânico por impacto. In: CONGRESSO BRASILEIRO DE FRUTICULTURA, 20., 2008, Vitória. Resumos... Vitória: SBF, 2008. p. 1. 
HENZ, G. P.; SOUZA, R. M.; PEIXOTO, J. R.; BLUMER, L. Danos causados pelo impacto de queda na qualidade pós-colheita de raízes de mandioquinha-salsa. Horticultura Brasileira, Brasília, v. 23, n. 4, p. 881-886, 2005.

KADER, A. A. Postharvest Biology and Tecnology: an overview. In: KADER, A. A.; KASMIRE, R. F.; MITCHELL, F. G.; REID, M. S.; SOMMER, N. F.; THOMPSON, J. F. (Ed.). Postharvest Technology of Horticultural Crops. Berkeley: University of California, 1985. p. 3-8.

LAU, O. L. Storage procedures, low oxygen and low carbon dioxide atmospheres on storage quality of 'Golden Delicious' and 'Delicious'apples. Journal of the American Society for Horticultural Science, Alexandria, v. 110, n. 4, p. 541-547, 1985.

MacLEOD, R. F.; KADER, A. A.; MORRIS, L. L. Damage to fresh tomatoes can be reduced. California Agriculture, Berkeley, v. 30, n. 12, p. 10-12, 1976.

MAO, L.; YING, T.; XI, Y.; ZHEN, Y. Respiration rate, ethylene production, and cellular leakage of fruit following vibrational stree HortScience, Alexandria, v. 30, n. 1, p. 145-149, 1995.

MATTIUZ, B.; DURIGAN, J. F. Efeito de injúrias mecânicas no processo respiratório e nos parâmetros químicos de goiabas "Paluma" e "Pedro Sato". Revista Brasileira de Fruticultura, Jaboticabal, v. 23, n. 2, p. 282-287, 2001.

MULLER, I. de; BRITO, R. M. de; BENDER, R. J. Instrumented Sphere for Compression Analysis. In: CONFERENCE INSTRUMENTATION AND MEASUREMENT TECHNOLOGY, 2008, Victoria. Proceedings... Victoria: [s.n.], 2008. p. 244.

PATTERSON, M. Handling granny smith apples: the biology and sotrage technology for maximum storage. In: FIFTH INTERNATIONAL CONTROLED
ATMOSPHER RESEARCH CONFERENCE, 5., 1989, Washington. Proceedings... Washington: [s.n.], 1989. p. 7.

PISARCZYK, J. M. Field harvest damage affects potato tuber respiration and sugar content. American Potato Journal, Madison, v. 59, n. 5, p. 205-211, 1982.

SALVEIT, M. E.; LOCY, R. D. Cultivar differences in ethylene production by wounded sweet potato roots. Journal of the American Society for Horticultural Science, Alexandria, v. 107, n. 6, p. 1114-1117, 1982.

SANCHES, J.; DURIGNA, J. F.; DURIGAN, M. F. B. Aplicação de danos mecânicos em abacates e seus efeitos na qualidade dos frutos. Engenharia Agrícola, Jaboticabal, v. 28, n. 1, p. 164-175, 2008.

SAQUET, A. A.; STREIF, J. Respiração e produção de etileno de maçãs armazenadas em diversas concentrações de oxigênio. Revista Brasileira de Agrociência, Pelotas, v. 8, n. 1, p. 71-75, 2002.

SAQUET, A. A.; STREIF, J.; BANGERTH, F. Changes in ATP, ADP and pyridine nucleotide levels related to the incidence of physiological disorders in 'Conference' pears and 'Jonagold' apples during controlled atmosphere storage. Journal of Science \& Biotechnology, Ashford, v. 75, n. 2, p. 243-249, 2000.

STEFFENS, C. A.; ESPÍNDOLA, B. P.; AMARANTE, C. V. T. do; SILVEIRA, J. P. G.; CHECHI, R.; BRACKMANN, A. Respiração, produção de etileno e qualidade de maçãs "Gala" em função do dano mecânico por impacto e da aplicação de 1-metilciclopropeno. Ciência Rural, Santa Maria, v. 38, n. 7, p. 1864-1870, 2008.

WILLS, R. H.; McGLASSON, W. B.; GRAHAM, D.; JOYCE, D. Postharvest: an introduction to physiology and handling of fruit, vegetables and ornamentals. Sidney: University of NSW Press, 1998. 262 p. 
